\title{
Educación quirúrgica en posgrado
}

\author{
Postgraduate surgical education
}

\author{
Jesús Tapia-Jurado ${ }^{*}$ y Yohevane García-Barrón² \\ ${ }^{1}$ Presidente de la Academia Mexicana de Cirugía, Coordinador de Cursos Quirúrgicos Avanzados de Posgrado; ${ }^{2}$ Coordinador de Cursos Quirúrgicos \\ Avanzados de Posgrado. Facultad de Medicina, UNAM, México
}

La cirugía nace con la propia humanidad, pero no es hasta los últimos 50 años cuando puede brindar a los pacientes procedimientos quirúrgicos menos traumáticos y más seguros. En el ámbito de la educación de los profesionales de la cirugía, es justo reconocer el trabajo que realiza la División de Estudios de Posgrado de la Facultad de Medicina de la Universidad Nacional Autónoma de México a través del PUEM' (Programa Único de Especializaciones Médicas), pensando siempre en el beneficio de los residentes y profesores, pero sobre todo de la sociedad mexicana, por lo que consideramos que debemos continuar colaborando instituciones educativas, de servicios de salud e instancias certificadoras, y que juntos analicemos nuestras inquietudes, deseos y experiencias, y así lograremos un nuevo momento de actualización del plan y programas de estudio de las diversas especialidades quirúrgicas.

Los avances en las ciencias básicas y clínicas han permitido la generación de nuevos conocimientos en apoyo al paciente quirúrgico, como son el manejo de la respuesta inflamatoria y metabólica del paciente quirúrgico grave, los diagnósticos más oportunos y precisos gracias a las tecnologías en imagenología y endoscopia, el apoyo nutricional parenteral, los avances en anestesia y ventilación, y los tratamientos farmacológicos (antibióticos, analgésicos, antitrombóticos); todo ello asociado a abordajes quirúrgicos menos traumáticos y más seguros, como son la cirugía de mínima invasión y la robótica. Lo anterior da por resultando la aparición de un gran acervo de conocimientos y habilidades que deben enseñarse en un tiempo limitado, lo que obliga a seleccionar qué, cómo, con qué profundidad y en qué momento deben ser enseñados y aprendidos los conocimientos y las habilidades manuales del futuro cirujano. Por tal motivo, en las residencias médicas la enseñanza debe estar centrada en las necesidades de los alumnos, las patologías a enfrentar de cada especialidad y las características de las unidades hospitalarias en donde laborarán. Además, debemos tomar en cuenta los conceptos neurobiológi$\cos ^{2}$ del aprendizaje, a saber, repetición del conocimiento mediante diversas estrategias hasta hacerlo reflexivo y crítico, repetición de las habilidades manuales hasta alcanzar la destreza, motivación, visualización de los conceptos, recompensa, estrés moderado y controlado que no lleve al agotamiento y la angustia, amplio compromiso personal, realización de multitareas sin dispersar la atención, entender que cada residente tiene un estilo diferente de aprender, reforzar la participación activa del binomio profesor-alumno, y hacer sistemática la revisión de los conocimientos mediante evidencias científicas. Por lo tanto, estamos obligados a ofrecer planes y programas de estudio actualizados, de vanguardia y que cubran las competencias profesionales indispensables para las necesidades en salud de la población mexicana (prevención, diagnóstico, tratamiento y rehabilitación de las patologías médico-quirúrgicas de cada una de las especialidades, además de brindar atención de la mejor calidad, seguridad y costo-beneficio), y que sean congruentes con las políticas asistenciales que requieren las instituciones de salud.

Por lo que toca a las estrategias de enseñanza, deben ser interactivas y entre ellas destacan el aprendizaje basado en problemas, la medicina basada en

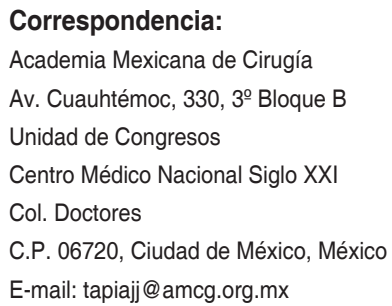

Cir Cir. 2018;86:125-127

Contents available at PubMed www.cirugiaycirujanos.com 
evidencias y resultados, la integración básico-clínica, las habilidades y destrezas quirúrgicas por simulación, y la educación a distancia. Para la teoría, se aconsejan sesiones dirigidas exclusivamente a médicos residentes de los diversos años; no estamos hablando de las sesiones obligatorias del hospital (clínico-quirúrgicas, anatomo-patológicas, de imagenología, manejo de tejidos, ética, etc., a las cuales también deben asistir), sino que estas son fuera del ámbito clínico-hospitalario, en pequeños grupos, en los que la oportunidad de participación del residente es amplia y completa, coordinados por su tutor o por el residente de mayor jerarquía, y tener discusiones clínicas, con énfasis en el razonamiento y la reflexión del diagnóstico, el tratamiento médico-quirúrgico, el pronóstico, la rehabilitación, el manejo de las complicaciones posoperatorias y los problemas éticos, sin olvidar que la clínica debe continuar en la cabecera del paciente, siendo la base del trabajo educativo. En cuanto a las habilidades médico-quirúrgicas, hay que identificar cuáles son las esenciales que el residente debe efectuar, con qué grado de dominio (novato, medio, experto) y si debe realizar un determinado número de cada procedimiento para alcanzar su expertis. Además, la práctica quirúrgica se debe ampliar en un ambiente que reproduzca la realidad en una forma controlada, cuya finalidad es adquirir o mejorar las competencias profesionales psicomotrices, de comunicación, coordinación y liderazgo. Estamos hablando de la estrategia educativa de la simulación ${ }^{3}$, que requiere cuatro etapas: cognitiva, integrativa, autónoma y de evaluación. La simulacion puede realizarse en modelos no biológicos y biológicos, y puede ser simple o compleja, barata o cara. Todas sus formas funcionan siempre y cuando tengamos claros los objetivos de aprendizaje a alcanzar. Actualmente se considera que la simulación es una necesidad ética, ya que a los pacientes se les protege al no ser objeto del aprendizaje. También el residente debe ser formado con fuerte apego al método científico, siempre preguntándose las causas de los fenómenos que alteran la salud, las hipótesis de trabajo y los resultados, lo que le llevará a una mejor toma de decisiones. Se deben realizar trabajos de investigación, que pueden ir de lo simple a lo complejo, desde una revisión de un caso clínico a encuestas epidemiológicas, trabajos comparativos y hasta estudios con gran número de casos, grupo control y aleatorizados. Siempre debemos influir al residente para que realice trabajos originales y evite el plagio o la repetición de información. El cirujano actual también debe aspirar a tener en su desarrollo curricular algún tipo de maestría o doctorado, que le permita desarrollar investigación médica clínica o experimental; por lo tanto, debemos insistir en la realización de una maestría en ciencias quirúrgicas.

Para lograr un perfil de egresado de calidad hay que incluir sistemas de evaluación ${ }^{4}$ que demuestren que el residente adquirió conocimientos y destrezas suficientes para ser competente en su profesión, y que mantendrá a lo largo de su vida una actitud de búsqueda del conocimiento (aprender a aprender) y de desarrollo profesional; ejemplos de ello son el Libro informático del residente quirúrgico en España, el eLogbook del Real Colegio de Cirujanos de Edimburgo en el Reino Unido, y los registros nacionales como el Practice-Based Learning System y el National Surgical Quality Improvement Program (NSQIP) que se utilizan en los EE.UU.

No olvidemos que para todo cirujano es fundamental mantener de por vida sus principios éticos y bioéticos, los cuales se deben reforzar o enseñar desde el principio de los estudios médicos. Por lo tanto, continúa siendo valioso fomentar las actitudes de los estudiantes en responsabilidad, honestidad, humildad, respeto, profesionalismo, autocrítica, humanismo, altruismo, discreción, tolerancia, liderazgo, solidaridad social y protección a su entorno y medio ambiente. Además, se deben reconocer y prevenir otros factores, como son la cirugía innecesaria, el cuidado quirúrgico en los últimos momentos de la vida y la implementación, el desarrollo y la adopción de nuevas tecnologías. En cuanto al proceso de enseñanza-aprendizaje del médico moderno, se menciona que debe ser científico, actualizado, humanístico y social, pero sobre todo ético ${ }^{5}$. Por lo que comentan algunos autores ${ }^{6}$, Ios principios a aplicar son:

- El aprendizaje debe estar centrado en las necesidades y las características del estudiante.

- El alumno debe contar con el tiempo suficiente para aprender y realizar lo enseñado.

- El alumno debe utilizar estrategias de aprendizaje adecuadas para retener lo aprendido.

- El alumno debe tener la oportunidad de explorar las consecuencias de su aprendizaje.

- Se debe dar oportunidad al alumno de practicar lo aprendido.

Para lograr estos grandes objetivos, el papel del profesor continúa siendo insustituible y debe seguir siendo el guía y tutor, el ejemplo a seguir y, por qué no, a superar. Especialistas como Perry y Edwards ${ }^{7}$ señalan que los docentes o tutores efectivos o ejemplares poseen las siguientes tres competencias fundamentales:

- Educadores como retadores (challengers), buscando siempre las más altas expectativas: ¡siempre puedes y puedes aún más! 
- Educadores como motivadores positivos (affirmers), alentando cada actividad y mostrando sus avances y reconociendo su propio potencial.

- Educadores como influyentes (influencers), lo que expresa una presencia fuerte y de gran experiencia en lo que enseña.

Por otro lado, es importante señalar que algunos residentes se quejan de exceso de trabajo, agotamiento y falta de sueño, lo que les lleva, según diversos reportes ${ }^{8}$, a un deficiente aprendizaje cognitivo y de habilidades manuales, alteraciones psicológicas, problemas maritales, el doble de accidentes automovilísticos en relación con la población normal, y generar errores médicos graves. Por ello, algunas organizaciones educativas internacionales han cambiado los horarios de trabajo de los residentes; el accreditation council for graduate medical education estableció, desde julio de 2003, un horario de trabajo de atención médico-quirúrgica no mayor de 80 horas a la semana y guardias de no más de 30 horas continuas, con posterior descanso de por lo menos 10 horas. Indudablemente, es complejo ponerse de acuerdo en este punto, ya que si bien es cierto que al disminuir los horarios de atención médico-quirúrgica se beneficia el tiempo de descanso de los residentes y se favorece una mejor calidad y seguridad en la atención médica de los pacientes, por otro lado se disminuye a los residentes las experiencias clínico-quirúrgicas de los pacientes hospitalizados.

Tampoco podemos ocultar el maltrato a los residentes, que algunos cirujanos mencionan como comportamiento habitual, tradicional y aceptable. Quine ${ }^{9}$, en el Reino Unido, menciona que el $84 \%$ de los médicos jóvenes han experimentado al menos un comportamiento de intimidación, y en América Latina las situaciones de acoso o maltrato a médicos residentes van del 71 al $89 \%$. En México ${ }^{10}$, el promedio de residentes que refirió haber sido al menos una vez víctima de maltrato, aunque fuera mínimo, fue del $52.87 \%$, siendo las especialidades quirúrgicas las que registran mayor frecuencia de conductas de violencia laboral, predominando en cirugía general, cirugía pediátrica y otorrinolaringología. Por lo tanto, es tiempo de que profesores y residentes acepten su responsabilidad y trasformen el maltrato en respaldo, confianza y solidaridad. Sin embargo, debemos mediar la balanza, ya que si bien es cierto que el residente se encuentra saturado de trabajo, agotado, con sueño y estresado, también es cierto que el trabajo del residente requiere orden, disciplina, gran compromiso y pasión.
Recordemos que, en ocasiones, a pesar de llevar todas las guías y normas pertinentes, nos encontramos que en la práctica quirúrgica se puedan presentar eventos adversos no previsibles, no prevenibles o inevitables. Por ello son motivo de gran preocupación los eventos adversos suscitados en medicina, y en particular en cirugía, hecho que sobresale a partir de la publicación del libro Errar es humano"11 en el año 2001. Por lo tanto, generar una conducta de calidad y seguridad es indispensable en la formación del profesional en salud.

Por todo lo anterior, consideramos que debemos trabajar unidos universidades e instituciones de salud para hacer frente a los retos que debemos superar en la educación quirúrgica, los cuales resumimos en los siguientes:

- Revisar, desarrollar y evaluar planes y programas de estudio que cubran las necesidades de conocimientos clínicos y de habilidades y destrezas quirúrgicas que requiere la sociedad mexicana, y que a la vez estén vinculados con los intereses de las instituciones de salud.

- Educar sobre principios éticos, bioéticos, de profesionalismo y liderazgo.

- Fomentar la investigación quirúrgica de vanguardia y traslacional.

- Favorecer la práctica quirúrgica basada en la cirugía segura.

- Mantener un desarrollo profesional continuo.

\section{Bibliografía}

1. Universidad Nacional Autónoma de México, México. Plan Único de Especializaciones Médicas (PUEM) en Cirugía General; c2009. (Consultado el 21 de septiembre de 2017.) Disponible en: http://www.sidep.fmposgrado.unam.mx:8080/fmposgrado/programas/cirgeneral.pdf

2. Friedlander MJ, Andrews L, Armstrong EG, Aschenbrenner C, Kass JS, Ogden $\mathrm{P}$, et al. What can medical education learn from the neurobiology of learning? Acad Med. 2011;86:415-20.

3. Tapia JJ, Vázquez A. Simulación como estrategia educativa en la adquisición de habilidades de destrezas quirúrgicas. En: Fenig RJ, editor. El entorno del cirujano general de principio a fin. Asociación Mexicana de Cirugía General, A.C. México; 2016. p. 131-6.

4. Escamirosa FP, Flores RMO, García IO, Vidal CRZ, Martínez AM. Face, content, and construct validity of the Endovis training system for objective assessment of psychomotor skills of laparoscopic surgeons. Surg Endosc. 2015;29:3392-403.

5. Fernández JM. Código de ética médica. Sociedad Mexicana de Ortopedia, A.C. México, D.F.; 2007. p. 109-11.

6. Guinchard SE. La ética en la formación del médico. Acta Ortopédica Mexicana. 2011;25:343-45.

7. Perry B, Edwards M. Exemplary online educators: creating a community of inquiry. Turkish Online Journal of Distance Education-TOJDE. 2005;6:46-54.

8. Mejía R, Diego A, Alemán M, Maliandi M, Lasala F. Percepción de maltrato durante la capacitación de médicos residentes. Medicina. 2005;65:366-8.

9. Quine L. Workplace bullying in junior doctors: questionnaire survey. BMJ. 2002;324:878-9.

10. Alcazar AL. Mobbing... Una manifestación de violencia en médicos residentes. Sesión conjunta con el Instituto de Seguridad y Servicios Sociales de los Trabajadores del Estado. México, D.F.: Academia Mexicana de Cirugía; 2017.

11. Makary M, Daniel M. Medical error - the third leading cause of death in the US. BMJ. 2016;353:i2139. 